\title{
ON THE EXISTENCE OF NORMAL SUBGROUPS CONTAINING THEIR CENTRALIZER
}

\author{
BY \\ ULRICH SCHOENWAELDER
}

Every finite $p$-group $G$ has a characteristic subgroup $N$ such that $G \circ N \subseteq{ }^{\prime} N$ $=\mathfrak{E}_{G} N$ with an elementary abelian factorgroup $N / \mathfrak{z} N$ (see W. Feit and J. G. Thompson [3, Lemma 8.2]). Every hyper-abelian group $G$ has a normal subgroup $N$ such that $N^{\prime} \subseteq{ }_{z} N=\mathfrak{\complement}_{G} N$ (see R. Baer [1, Lemma 4.1]). The proof of Baer's lemma is applicable in much more general situations which can best be described by what we call subgroup theoretical properties. From a general theorem we shall not only derive both of the above mentioned lemmas but also a variety of similar results. Here we mention only the following generalization of the Feit-Thompson lemma and of the case $\pi=\{p\}$ of a result of P. Hall and G. Higman ([4, Lemma 1.2.3]):

For every abelian, characteristic subgroup $M$ of a p-solvable, finite group $G$ whose only normal $p^{\prime}$-subgroup is 1 , there exists a characteristic p-subgroup $N$ of $G$ with $M \subseteq{ }_{z} N=\mathfrak{\complement}_{G} N$ and $N / z N \subseteq \Omega_{1 子}\left(\mathfrak{D}_{p} G / z N\right.$ ) (see $\S 6$. VII).

I am greatly indebted to Professor R. Baer for many remarks including an extension of his lemma to certain almost hyper-abelian groups which suggested the introduction of the property $\beta$ below.

Notation will be standard with $\langle X, Y\rangle$ denoting the subgroup generated by $X$ and $Y$.

1. Statement of main theorem. Throughout the paper a group $G$ will be fixed and $\nu$ will be a set of subgroups of the group $G$, called $\nu$-subgroups, such that:

(1) 1 is a $\nu$-subgroup.

(2) $X, Y \in \nu$ implies $\langle X, Y\rangle \in \nu$.

A subgroup theoretical property on $\nu$ is a set of triples $(X, Y, G)$ of $\nu$-subgroups $X \subseteq Y$ of $G$. We say that the subgroup theoretical property $\alpha$ is derived from the group theoretical property $e$ if $\nu$ is the set of all normal subgroups of $G$ and $\alpha$ as a subgroup theoretical property on $v$ is characterized by

$$
(X, Y, G) \in \alpha \text { if and only if } Y / X \text { is an } e \text {-group. }
$$

The following conditions will be placed on $\nu$, subgroup theoretical properties $\alpha$ and $\beta$ on $\nu$, and a $\nu$-subgroup $R$ of $G$ in the main theorem:

(3) $X, Y \in \nu$ implies $X \cap Y \in \nu$.

(4) The union of a tower of $\nu$-subgroups is a $\nu$-subgroup.

Received by the editors April 3, 1969. 
(5) $(Z, X, G) \in \alpha,(Z, Y, G) \in \alpha, Z=X \cap Y$ implies $(Z,\langle X, Y\rangle, G) \in \alpha$.

(6) For $\nu$-subgroups $J$ and $Y$ the following statements are equivalent:

(i) $(J \cap Y, Y, G) \in \alpha$,

(ii) $(J,\langle J, Y\rangle, G) \in \alpha$,

(iii) there exists a $\nu$-subgroup $L \supseteq\langle J, Y\rangle$ with $(J, L, G) \in \alpha$.

(7) $A, C \in \nu, R \cap C \subseteq A \subseteq C,(R, G, G) \in \beta$ implies $(A, C, G) \in \beta$.

For a discussion of these conditions see $\S \S 3$ and 4 .

Definition. Let $R$ be a $\nu$-subgroup of $G$ and $\alpha$ a subgroup theoretical property on $\nu$. We say $R$ is $[\nu, \alpha]$-immersed in $G$, in symbols $R[\nu, \alpha] G$, if

(8) $R \nsubseteq K \in \nu$ implies that there exists a $\nu$-subgroup $D$ with $K \subset D \subseteq\langle K, R\rangle$ and $(K, D, G) \in \alpha$.

Therefore, if $\alpha$ is derived from the group theoretical property $e, G[\nu, \alpha] G$ if and only if $G$ is a hyper-e group.

Definition. A centralizer function $c$ on $v$ is a single valued mapping from $v$ to $v$ such that after introducing the $c$-center $c_{0} X=X \cap c X$ for $X \in \nu$ the following condition is satisfied:

(9) $N, B \in \nu, N \cap B=c_{0} N \subseteq B \subseteq c N$ implies $c_{0} N \subseteq c\langle N, B\rangle$.

Note that by definition $c X$ is always a $\nu$-subgroup for $X \in \nu$.

We are now in a position to state the main theorem.

THEOREM. Let $\alpha$ and $\beta$ be subgroup theoretical properties on $\nu$ and $R$ a $\nu$-subgroup of $G$ such that (1)-(7) are satisfied. Suppose $R[\nu, \alpha] G$ and $(R, G, G) \in \beta$. Let c be a centralizer function on $v$ and assume that there exist $\nu$-subgroups $A \subseteq N$ such that $A$ is maximal among the $c_{0} \nu$-subgroups and $N$ is maximal among the $\nu$-subgroups $T$ with $A=c_{0} T$ and $(A, T, G) \in \alpha$. Then $\left(c_{0} N, c N, G\right) \in \beta$.

The proof appears in the next section.

2. The abstract situation. We break up the proof of the theorem into several steps.

Proposition 1. Let $\alpha$ and $\beta$ be subgroup theoretical properties on $\nu$ and $c$ a centralizer function on $\nu$. Assume that there exist $\nu$-subgroups $A \subseteq N$ of $G$ such that $A$ is maximal among the $c_{0} \nu$-subgroups and $N$ is maximal among the $\nu$-subgroups $T$ with $A=c_{0} T$ and $(A, T, G) \in \alpha$. Let (5) be satisfied by $Z=A, X=N$ and suppose

(10) if $A \subseteq C \in \nu$ and $(A, C, G) \notin \beta$, then there exists a $\nu$-subgroup $B$ with $A \subset B \subseteq C$ and $(A, B, G) \in \alpha$.

Then $\left(c_{0} N, c N, G\right) \in \beta$.

Proof. $A=c_{0} N$ by choice of $N . c$ being a centralizer function on $\nu, C=c N$ is a $\nu$-subgroup. Assume by way of contradiction that $(A, C, G) \notin \beta$. Then by $(10)$, there exists a $\nu$-subgroup $B$ with $A \subset B \subseteq C$ and $(A, B, G) \in \alpha$. We have $A \subseteq N \cap B$ $\subseteq N \cap c N=A$, hence $A=N \cap B$, and (5) implies $(A,\langle N, B\rangle, G) \in \alpha$. Since $c$ is a centralizer function we get $A=c_{0} N \subseteq c\langle N, B\rangle$. So $A \subseteq\langle N, B\rangle \cap c\langle N, B\rangle=c_{0}\langle N, B\rangle$ 
and $A=c_{0}\langle N, B\rangle$ by the maximality of $A$. But this implies $N=\langle N, B\rangle$ by the maximality of $N$. Hence $A \subset B \subseteq N \cap c N=A$, q.e.a.

Lemma. (See R. Baer [2, Proposition 1.5].) Let $\alpha$ be a subgroup theoretical property on $\nu$ such that (1)-(4) and (6) are satisfied. Let $S \subseteq R$ be $\nu$-subgroups of $G$. Then $R[\nu, \alpha] G$ implies $S[\nu, \alpha] G$.

Proof. Let $K$ be a $\nu$-subgroup that does not contain $S$. Then $S \cap K \subset S$. Since $\nu$ satisfies the tower condition (4) there exists, by Zorn's lemma, a subgroup $J$ of $G$ maximal among the subgroups $X$ of $G$ with

$$
K \subseteq X \in \nu, \quad S \cap K=S \cap X .
$$

If $R \subseteq J$, then $S \subseteq J$ and $S=S \cap J=S \cap K \subset S$, q.e.a. So $R \nsubseteq J$. By hypothesis, $R$ is $[\nu, \alpha]$-immersed in $G$ and therefore there exists a $\nu$-subgroup $L$ with

$$
J \subset L \subseteq\langle J, R\rangle \quad \text { and } \quad(J, L, G) \in \alpha,
$$

and by the maximality of $J$ we also have $S \cap K \subset S \cap L$. Put $Y=S \cap L$ and $D=\langle K, Y\rangle$ so that $K \subset D \subseteq\langle K, S\rangle$. D is a $\nu$-subgroup. Moreover, $(J, L, G) \in \alpha$ implies $(J \cap Y, Y, G) \in \alpha$ by the implication (iii) $\rightarrow$ (i) in (6). But $J \cap Y=J \cap S$ $=K \cap S=K \cap Y$ so that $(K \cap Y, Y, G) \in \alpha$ and $(K, D, G)=(K,\langle K, Y\rangle, G) \in \alpha$ by (6). This proves that $S$ is $[\nu, \alpha]$-immersed in $G$.

Proposition 2. Let $\alpha$ and $\beta$ be subgroup theoretical properties on $v$ such that (1)-(4) and (6) are satisfied. Suppose that $R$ is a $[v, \alpha]$-immersed $v$-subgroup of $G$ with $(R, G, G) \in \beta$. Then (7) implies (10) for all $\nu$-subgroups $A$.

Proof. Let $A \subseteq C$ be $\nu$-subgroups with $(A, C, G) \notin \beta$. Then (7) implies $R \cap C \nsubseteq A$. By the lemma $R \cap C$ is $[\nu, \alpha]$-immersed and therefore there exists a $\nu$-subgroup $B$ with $A \subset B \subseteq\langle A, R \cap C\rangle \subseteq C$ and $(A, B, G) \in \alpha$.

Proof of Theorem. By Proposition 2, Proposition 1 is applicable.

3. Examples for properties $\alpha$ satisfying (5) and (6). If $\nu$ consists of normal subgroups of $G$ only then subgroup theoretical properties $\alpha$ on $\nu$ with (5) and (6) may be defined as follows:

I. For a group theoretical property $e$ inherited by normal subgroups and direct products and $\nu$-subgroups $A \subseteq B$ let $(A, B, G) \in \alpha$ if and only if $B / A$ is an $e$-group.

Proof. A group isomorphic to an $e$-group is an $e$-group.

II. For a group theoretical property $h$ that is inherited by subgroups, epimorphic images and direct products, and $\nu$-subgroups $A \subseteq B$ let $(A, B, G) \in \alpha$ if and only if $G / \mathfrak{C}_{G}(B \bmod A)$ is an $h$-group.

Proof. (5) follows from the fact that $G / \mathfrak{S}_{G}(X Y \bmod Z)$ is isomorphic to a subgroup of the direct product of $G / \mathbb{E}_{G}(X \bmod Z)$ with $G / \mathbb{S}_{G}(Y \bmod Z)$. In (6), (i) and (ii) are equivalent since $\mathfrak{C}_{G}(Y \bmod J \cap Y)=\mathfrak{C}_{G}(J Y \bmod J)$, and (iii) implies (ii) because of $\mathfrak{夭}_{G}(L \bmod J) \subseteq \mathfrak{夭}_{G}(J Y \bmod J)$. 
III. Let $\alpha=\alpha_{1} \cap \alpha_{2}$ for subgroup theoretical properties $\alpha_{1}$ and $\alpha_{2}$ that satisfy (5) and (6) for the same set $\nu$.

4. Examples for properties $\beta$ satisfying (7). If $\nu$ consists of normal subgroups of $G$ only and if $R$ is a $\nu$-subgroup of $G$, then subgroup theoretical properties $\beta$ which satisfy (7) may be defined as follows:

I. For a group theoretical property $g$ that is inherited by normal subgroups and epimorphic images, and $\nu$-subgroups $X \subseteq Y$ let $(X, Y, G) \in \beta$ if and only if $Y / X$ is a $g$-group.

Proof. Under the hypothesis of (7) $R Y / R$ as a normal subgroup of $G / R$ is a $g$-group. So $C / A$ is a $g$-group as an epimorphic image of $C / R \cap C \cong R C / R$.

In many an application $g$ will be the group theoretical property 1 (and $R=G$ ).

II. For $\nu$-subgroups $X \subseteq Y$ let $(X, Y, G) \in \beta$ if and only if

$$
\mathfrak{z}(G \bmod R) \subseteq \mathfrak{S}_{G}(Y \bmod X) .
$$

Proof. Under the hypothesis of (7) with $Z=z(G \bmod R)$ we have

$$
Z \circ C \subseteq(Z \circ G) \cap C \subseteq R \cap C \subseteq A,
$$

hence $Z \subseteq \mathfrak{\subseteq}_{G}(C \bmod A)$ and $(A, C, G) \in \beta$.

Note that with this $\beta$ always $(R, G, G) \in \beta$ so that in the Theorem we always get $Z \subseteq \mathfrak{C}_{G}\left(c N \bmod c_{0} N\right)$. In this view the following example becomes useful.

III. Let $\beta=\beta_{1} \cap \beta_{2}$ for subgroup theoretical properties $\beta_{1}$ and $\beta_{2}$ that satisfy (7) for the same set $\nu$.

5. Examples for centralizer functions. All examples below give centralizer functions of a special type arising as follows:

5.1. Let $\sigma$ be a relation on $\nu$ such that:

(a) the intersection of two $\nu$-subgroups is a $\nu$-subgroup (3),

(b) every set of $\nu$-subgroups generates a $\nu$-subgroup,

(c) if $N \in \nu$ and if $\mathfrak{X}$ is a set of $\nu$-subgroups $X$ with $X \sigma N$, then $\langle\mathfrak{X}\rangle \sigma N$,

(d) $U, X, N \in \nu, U \subseteq X \sigma N$ implies $U \sigma N$,

(e) $U, V \in \nu, U \sigma V$ implies $V \sigma U$.

For $N \in \nu$ define $c N=\langle X \mid X \in \nu, X \sigma N\rangle$. Then $c$ is a centralizer function on $\nu$.

Proof. By (b), $c$ is a single valued mapping from $\nu$ to $\nu$. To check (9), let $N, B \in \nu$ with $B \subseteq c N$. Then $c N \sigma N$ by (c), $N \cap c N \in \nu$ by (a), and $N \cap c N \sigma N$ by (d). Also $B \sigma N$ by (d), $N \sigma B$ by (e), and $N \cap c N \sigma B$ by (d). Hence $N \cap c N \sigma\langle N, B\rangle$ by (e) and (c). Therefore $c_{0} N \subseteq c\langle N, B\rangle$.

5.2. The theorem requires the existence of maximal $c_{0} \nu$-subgroups. Since $1 \in \nu$ by (1), there always exist $c_{0} v$-subgroups, e.g. $c_{0} 1=1$. So if

(11) the union of a tower of $c_{0} \nu$-subgroups is a $c_{0} v$-subgroup, then by the Maximum Principle of Set Theory, maximal $c_{0} \nu$-subgroups do exist. We shall check the validity of (11) in the following examples for 5.1.

Suppose that $\nu$ satisfies (1), 5.1(a), and 5.1(b). 
I. For $U, V \in \nu$ let $U \sigma V$ if and only if $U$ and $V$ centralize each other. Then (a)-(e) in 5.1 are satisfied. If $\mathfrak{夭}_{G} \nu \subseteq \nu$, then $c N$ becomes the centralizer of $N$ in $G$. In general however, $c N$ may be smaller than $\Im_{G} N$. By 5.1(a), $c_{0} \subseteq \subseteq \nu$. A $\nu$-subgroup is a $c_{0} \nu$-subgroup if and only if it is abelian. So by 5.1(b), $c_{0} \nu$ satisfies the tower condition (11).

II. For $U, V \in \nu$ let $U \boldsymbol{\sigma} V$ if and only if every subgroup of $U$ permutes with every subgroup of $V$. To prove (c) in 5.1 let $t \in\langle\mathfrak{X}\rangle, n \in N$. Then $t$ is a product of elements $x$ in various $X \in \mathfrak{X}$. But $x n=\bar{n} \bar{x}$ for some $\bar{n} \in\langle n\rangle \subseteq N, \bar{x} \in\langle x\rangle \subseteq X$, since $X \sigma N$. So $t n=\tilde{n} \tilde{t}$ with $\tilde{n} \in\langle n\rangle \subseteq N, \tilde{t} \in\langle\mathfrak{X}\rangle$ proving (c). (d) and (e) are obvious. By $5.1, \mathfrak{B} N=c N=\langle X \in \nu \mid X \sigma N\rangle$ defines a centralizer function $c=\mathfrak{B}$ on $\nu$. A $\nu$-subgroup $Z$ of $G$ is a $c_{0} \nu$-subgroup if and only if $Z \sigma Z$. It is now easy to see that 5.1(b) implies the tower condition (11). Finite $c_{0} v$-subgroups are nilpotent (M. Suzuki [6, p. 7]).

III. Let $\mathfrak{A}$ be a group of automorphisms of $G$ that contains all inner automorphisms and suppose that $\nu$ consists of $\mathfrak{A}$-invariant subgroups only and satisfies (3) (e.g. the set of all $\mathfrak{A}$-invariant subgroups meets requirement (3)). An $\mathfrak{A}$-composition factor $U_{1} \mathfrak{A} U_{2}$ of $G$ contained in $U \subseteq G$ will be a pair $\left(U_{1}, U_{2}\right)$ of $\mathfrak{A}$-invariant subgroups $U_{2} \subseteq U_{1} \subseteq U$ with no other $\mathfrak{A}$-invariant subgroup between them. $U_{1} \mathfrak{A} U_{2}$ centralizes $V_{1} \mathfrak{A} V_{2}$ if $U_{1} \circ V_{1} \subseteq U_{2} \cap V_{2}$.

For $U, V \in \nu$ let $U \sigma V$ if and only if the $\mathfrak{A}$-composition factors of $G$ contained in $U$ centralize the $\mathscr{U}$-composition factors of $G$ contained in $V$. To prove $5.1(\mathrm{c})$ let $Z_{1} \mathfrak{A} Z_{2}$ be an $\mathfrak{A}$-composition factor in $Z=\langle\mathfrak{X}\rangle$ and $N_{1} \mathfrak{A} N_{2}$ an $\mathfrak{A}$-composition factor in $N$. Consider first the case where $\mathfrak{X}$ has just two members $X$ and $Y$.

Clearly

$$
Z_{1}=Z_{2}\left(Z_{1} \cap X\right) \text { or } Z_{1} \cap X \subseteq Z_{2} \text {, }
$$

since $Z_{1} \mathfrak{A} Z_{2}$ is an $\mathfrak{A}$-composition factor and $Z_{2}\left(Z_{1} \cap X\right)$ is $\mathfrak{A}$-invariant. In the first case

$$
Z_{1} \circ N_{1} \subseteq\left[Z_{2} \circ N_{1}\right]\left[\left(Z_{1} \cap X\right) \circ N_{1}\right] \subseteq Z_{2}\left(Z_{2} \cap X\right) \subseteq Z_{2},
$$

since $\left(Z_{1} \cap X\right) \mathfrak{U}\left(Z_{2} \cap X\right)$ is an $\mathfrak{A}$-composition factor in $X \sigma N$. Also $X Z_{1} \mathfrak{A} X Z_{2}$ and $\left(X Z_{1} \cap Y\right) \mathfrak{A}\left(X Z_{2} \cap Y\right)$ are $\mathfrak{A}$-composition factors, and

$$
X Z_{1}=\left(X Z_{2}\right) Z_{1}=X Z_{2}\left(X Z_{1} \cap Y\right) .
$$

Hence

$$
X Z_{1} \circ N_{1} \subseteq\left[X Z_{2} \circ N_{1}\right]\left[\left(X Z_{1} \cap Y\right) \circ N_{1}\right] \subseteq X Z_{2}\left(X Z_{2} \cap Y\right) \subseteq X Z_{2}
$$

so that in the second case

$$
Z_{1} \circ N_{1} \subseteq Z_{1} \cap\left(X Z_{1} \circ N_{1}\right) \subseteq Z_{1} \cap X Z_{2}=Z_{2}\left(Z_{1} \cap X\right) \subseteq Z_{2} .
$$

Clearly $Z_{1} \circ N_{1} \subseteq X Y \circ N_{1} \subseteq\left[X \circ N_{1}\right]\left[Y \circ N_{1}\right] \subseteq N_{2}$ since $X \mathfrak{A} X$ and $Y \mathfrak{A} Y$ are $\mathfrak{A}$-composition factors. This proves $X Y \sigma N$.

In the general case there exists a $\nu$-subgroup $W$ of $\langle\mathfrak{X}\rangle$ maximal with $W \sigma N$ by 
the Maximum Principle of Set Theory and 5.1(b); the argument is similar to the one given below for the existence of maximal $c_{0} \nu$-subgroups. Let $X \in \mathfrak{X}$. Then $W X \sigma N$ by the special case, hence $X \subseteq W$ by the maximality of $W$. Therefore $\langle\mathfrak{X}\rangle=W \sigma N$ proving (c). (d) and (e) follow immediately. By 5.1, $c N=\mathfrak{\Re} N=$ $\langle X \mid X \in \nu, X \sigma N\rangle$ defines a centralizer function $c=\mathfrak{\Omega}$ on $\nu$.

Let $\mathfrak{I}$ be a tower of $c_{0} \nu$-subgroups $Z$. Every $Z \in \mathfrak{T}$ is a $\nu$-subgroup with $Z \sigma Z$. By 5.1(b), $W=\bigcup \mathfrak{T} \in \nu$. For an $\mathfrak{A}$-composition factor $W_{1} \mathfrak{A} W_{2}$ of $G$ in $W$ let $w_{1} \in W_{1}$ and $w \in W$. Then there exists $Z \in \mathfrak{T}$ which contains both $w_{1}$ and $w$. Moreover $\left(W_{1} \cap Z\right) \mathfrak{A}\left(W_{2} \cap Z\right)$ is an $\mathfrak{A}$-composition factor in $Z$. Therefore $w_{1} \circ w \in W_{2} \cap Z$ showing that $W_{1} \circ W \subseteq W_{2}$ and hence that $W \sigma W$. (11) holds.

5.3. Let $\nu$ satisfy (1) and 5.1(b) and let $f$ be a function from $\nu$ into a set $\bar{\nu}$ of subgroups of $G$. Suppose that $\bar{\nu}$ and a relation $\bar{\sigma}$ on $\bar{\nu}$ satisfy (1) and 5.1(b)-(e). Then the relation $\sigma$ on $\nu$ defined by

\section{$U \sigma V$ if and only if $f U \bar{\sigma} f V$}

satisfies $5.1(\mathrm{c})-(\mathrm{e})$ provided that

(i) If $\mathfrak{X}$ is a set of $\nu$-subgroups, then $f\langle\mathfrak{X}\rangle=\langle f \mathfrak{X}\rangle$.

So if 5.1(a) holds for $\nu$ and $\bar{\nu}$, we have a centralizer function $c$ on $\bar{\nu}$ defined by $\bar{\sigma}$ and a centralizer function $c[f]$ on $\nu$ defined by $\sigma$.

Proof. For a set $\mathfrak{X}$ of $\nu$-subgroups $X$ with $X \sigma N$, i.e. $f X \bar{\sigma} f N$, we get $\langle f \mathfrak{X}\rangle \bar{\sigma} f N$ by (c) for $\bar{\nu}$ and $\bar{\sigma}$. Therefore $f\langle\mathfrak{X}\rangle \bar{\sigma} f N$ by (i), i.e. $\langle\mathfrak{X}\rangle \sigma N$, proving (c) for $\nu$ and $\sigma$. (d) follows from

(ii) $U, V \in \nu, U \subseteq V$ implies $f U \subseteq f V$, which is a consequence of (i). (e) is obvious.

5.4. Let $\gamma$ be a set of triples $(X, Y, G)$ with $X \in \nu, Y \in \bar{\nu}$. Note, however, that we do $n o t$ require $X \subseteq Y$. For $X \in \nu$ let

$$
\gamma X=G \cap \bigcap\{T \mid(X, T, G) \in \gamma\} .
$$

We make the following assumptions:

( $\gamma$.a) $\nu$ satisfies (1), 5.1(a), and 5.1(b).

( $\gamma$. b) If $\mathfrak{Y}$ is a set of $\bar{\nu}$-subgroups $Y$ with $(X, Y, G) \in \gamma$, then $(X, G \cap \cap \mathfrak{Y}, G) \in \gamma$.

( $\gamma$.c) $X_{1} \in \nu, X_{1} \subseteq X_{2},\left(X_{2}, Y, G\right) \in \gamma$ implies $\left(X_{1}, Y, G\right) \in \gamma$.

( $\gamma$.d) If $\mathfrak{X}$ is a set of $\nu$-subgroups, then $(\langle\mathfrak{X}\rangle,\langle\gamma \mathfrak{X}\rangle, G) \in \gamma$.

Then $(X, \gamma X, G) \in \gamma$ for $X \in \nu$ by $(\gamma . \mathrm{b})$, and $\gamma\langle\mathfrak{X}\rangle \subseteq\langle\gamma \mathfrak{X}\rangle$ by $(\gamma \cdot \mathrm{d})$. Let $(\langle\mathfrak{X}\rangle, T, G)$ $\in \gamma$. Then $(X, T, G) \in \gamma$ for all $X \in \mathfrak{X}$ by $(\gamma . \mathrm{c})$, hence $\gamma X \subseteq T$ and $\langle\gamma \mathfrak{X}\rangle \subseteq T$. Therefore $\langle\gamma \mathfrak{X}\rangle \subseteq \gamma\langle\mathfrak{X}\rangle$. This shows that

$$
f X=\gamma X
$$

satisfies (i) in 5.3. The following functions $f$ arise in this manner:

I. If every $\nu$-subgroup of $G$ is a normal subgroup of $G$, and if $\nu$ satisfies (1), 5.1(a), and 5.1(b), then a function $f$ with property (i) is defined by

$$
f X=X \circ G \text { for } X \in \nu, \quad \bar{\nu}=\text { set of all normal subgroups of } G .
$$


$f$ may be obtained from the set $\gamma$ defined by

$$
(X, Y, G) \in \gamma \quad \text { if and only if } X \in \nu, Y \in \bar{\nu}, X \circ G \subseteq Y .
$$

If $c$ is a centralizer function on $\bar{\nu}$ defined by a relation $\bar{\sigma}$ according to 5.1 , then

$$
c[f] N=\langle X \mid X \in \nu, X \circ G \bar{\sigma} N \circ G\rangle .
$$

In particular,

$$
\mathfrak{\wp}_{G}[f] N=\mathfrak{z}\left(G \bmod \mathfrak{夭}_{G}(N \circ G)\right) \text { for } N \in \nu
$$

provided that $\mathrm{z}\left(G \bmod \mathfrak{夭}_{G}(N \circ G)\right)$ is a $\nu$-subgroup.

II. Let $\nu$ satisfy (1), 5.1(a), and 5.1(b) and let $\bar{\nu}$ be the set of all $\mathfrak{A}$-invariant subgroups of $G$ for a fixed group $\mathfrak{A}$ of automorphisms of $G$. Then

$$
(X, Y, G) \in \gamma \quad \text { if and only if } X \in \nu, Y \in \bar{\nu} \text {, and } X \subseteq Y
$$

defines a set of triples with properties $(\gamma \cdot \mathrm{a})-(\gamma \cdot \mathrm{d})$ and consequently a function $f$ with property (i); $f X=\gamma X$ is the $\mathfrak{A}$-invariant hull of the $\nu$-subgroup $X$.

III. One may derive functions $f$ from suitable group theoretical properties $e$ via

$$
(X, Y, G) \text { if and only if } X, Y \unlhd G, X \subseteq Y, G / Y \text { is an e-group. }
$$

However, the resulting centralizer functions $\mathfrak{c}_{G}[f]$ do not lead to interesting applications in connection with the theorem.

\section{Some applications of the theorem.}

I. (R. Baer [1].) For every abelian, normal subgroup $M$ of a hyperabelian group $G$ there is a normal subgroup $N$ of $G$ with $M \subseteq{ }_{z} N=\mathfrak{C}_{G} N$ and $N^{\prime} \subseteq z_{z} N$.

Proof. We let $\nu$ be the set of all normal subgroups of $G$. The group theoretical property $e$ of being abelian is inherited by normal subgroups and direct products. Hence the subgroup theoretical property $\alpha$ derived from $e$ satisfies (5) and (6) (see 3.I). $R=G$ satisfies (8) by definition of "hyperabelian". $\beta$ may be defined as in 4.I with $g=1$ so that (7) holds trivially. There exists a maximal $z \nu$-subgroup $A \supseteq M$ as indicated in 5.2.I and there exist subgroups $N$ maximal among the normal subgroups of $G$ with $N^{\prime} \subseteq A={ }_{z} N$. By the theorem $z N=\mathfrak{C}_{G} N$.

II. For every abelian, normal subgroup $M$ of a finite, solvable group $G$ there is a normal subgroup $N$ of $G$ with $M \subseteq{ }_{z} N=\mathfrak{C}_{G} N$ and $N / z=$ abelian of squarefree exponent.

Proof as in I where, however, e-groups are abelian of squarefree exponent.

Since $N$ is nilpotent this result implies that the fitting subgroup of a solvable group contains its centralizer.

III. Let $\nu$ be the set of all normal subgroups of the group $G, g$ a group theoretical property that is inherited by normal subgroups and epimorphic images, and $\alpha$ the subgroup theoretical property derived from $e=$ abelian as in I. Suppose that $G$ has a $[\nu, \alpha]$-immersed normal subgroup $R$ whose factor group $G / R$ is a g-group. Then for 
every abelian, normal subgroup $M$ of $G$ there exists a normal subgroup $N$ of $G$ with the following properties:

$$
\begin{aligned}
& M \subseteq z_{z} N, \\
& N / z N \text { is abelian, } \\
& \mathfrak{夭}_{G} N / z N \text { is a } g \text {-group, } \\
& \mathfrak{夭}_{G}\left(\mathfrak{\mho}_{G} N \bmod { }_{z} N\right) \supseteq z(G \bmod R) .
\end{aligned}
$$

Proof. Define $\beta_{1}$ as $\beta$ in 4.I, $\beta_{2}$ as $\beta$ in 4.II, and $\beta=\beta_{1} \cap \beta_{2}$ as in 4.III so that $(R, G, G) \in \beta$ and (7). $\nu$ satisfies (1)-(4) and $\alpha$ meets requirements (5) and (6) as pointed out in 3.I. As in the proof of 6.I there are normal subgroups $A$ and $N$ with $M \subseteq A$ and the properties mentioned in the main theorem, namely

$$
A={ }_{z} N, \quad(A, N, G) \in \alpha, \quad\left(\mathfrak{z} N, \mathfrak{\complement}_{G} N, G\right) \in \beta .
$$

This means that $M \subseteq z N, N / z=$ is abelian, $\mathfrak{夭}_{G} N / \mathfrak{z} N$ is a $g$-group, and

$$
\mathfrak{夭}_{G}\left(\mathfrak{夭}_{G} N \bmod { }_{z} N\right) \supseteq \mathfrak{z}(G \bmod R) .
$$

In III, $\nu, \alpha$ and $\delta=\beta_{1}$ satisfy the hypothesis of Proposition 4 in $\$ 7$ below. Therefore, if every epimorphic image of $G$ is a $g$-group or has an abelian, normal subgroup, not 1 , then the $[\nu, \alpha]$-hypercenter $\mathfrak{h}_{[v, \alpha]} G$ as defined in $\S 7$ has the properties imposed on $R$ above.

Of course, 6.I is a special case of III.

IV. Let $G$ have a $[\nu, \alpha]$-immersed, normal subgroup $R$ as in III whose factor group $G / R$ is abelian. Then there exists for every abelian, normal subgroup $M$ of $G$ a normal subgroup $N$ of $G$ with $M \cdot N^{\prime} \cdot\left(G \circ \mathfrak{\complement}_{G} N\right) \subseteq{ }_{z} N$.

Proof. This is a special case of III.

V. For every characteristic, abelian subgroup $M$ of a hypercentral group $G$ there is a characteristic subgroup $N$ with $M(N \circ G) \subseteq{ }_{z} N=\complement_{G} N$.

Proof. Let $\nu$ be the set of all characteristic subgroups of $G$ and let $\alpha$ be defined as in 3.II with $h=1$. Then $R=G$ is $[\nu, \alpha]$-immersed in $G$ by the definition of "hypercentral", and the main theorem is applicable.

VI. (W. Feit and J. G. Thompson [3].) For every abelian, characteristic subgroup $M$ of a finite p-group $G$ there is a characteristic subgroup $N$ of $G$ with $M(N \circ G)$ $\subseteq{ }_{z} N=\mathfrak{C}_{G} N$ and elementary abelian factor group $N /{ }_{z} N$.

Proof. As for V. Special case of VII below.

REMARK. In the situation of $\mathrm{V}$ and VI, the (characteristic) subgroup $A$ in the main theorem uniquely determines a subgroup $N$ as described in the theorem. In the notation of [5], $N=\mathfrak{X}_{G}(A)$. However, the generalizations of $\mathrm{V}$ and VI contained in [5] though of a similar formal nature do not fit in our present context and certainly cannot be expected to follow from such a simple observation as Proposition 1 in $\$ 2$. 
VII. Result on finite, p-solvable groups as stated in the introduction.

Proof. Let $\nu$ be the set of all characteristic subgroups of $G$ and define the subgroup theoretical property $\alpha$ on $\nu$ by

$$
(X, Y, G) \in \alpha \quad \text { if and only if } Y / X=Y_{1} / X \otimes Y_{2} / X
$$

with a $p^{\prime}$-group $Y_{1} / X$ and an elementary abelian $p$-group $Y_{2} / X$ that centralizes $\mathfrak{D}_{p} G$.

We have to show that $G$ is $[v, \alpha]$-immersed in itself and that $\alpha$ satisfies (5) and (6). Let $K \neq G$ be a characteristic subgroup of $G$. Since $G$ is $p$-solvable,

$$
\mathfrak{D}_{p^{\prime}}(G / K) \otimes \mathfrak{D}_{p}(G / K) \neq 1 \text {. }
$$

If $\mathfrak{D}_{p^{\prime}}(G / K) \neq 1$, let $D / K=\mathfrak{D}_{p^{\prime}}(G / K)$. $\mathfrak{D}_{p} G$ induces a $p$-group of automorphisms of $\mathfrak{D}_{p}(G / K)$. So if $\mathfrak{D}_{p^{\prime}}(G / K)=1$ then $\mathfrak{D}_{p}(G / K) \neq 1$ and the fixed elements in $\mathfrak{D}_{p}(G / K)$ under the action of $\mathfrak{D}_{p} G$ form a subgroup $F / K \neq 1$; it is invariant under all automorphisms of $G$ and so is $D / K=\Omega_{1} z(F / K) \neq 1 ; D$ is a characteristic subgroup of $G$ and $D / K$ is elementary abelian and centralizes $\mathfrak{S}_{p} G$. Therefore $D$ is in both cases a characteristic subgroup of $G$ with $K \subset D$ and $(K, D, G) \in \alpha$. This proves $G[\nu, \alpha] G$.

One checks easily that $\alpha$ meets requirements (5) and (6).

Thus, $G$ has a characteristic subgroup $N$ with $M \subseteq z N=\mathbb{E}_{G} N$ and $(z N, N, G) \in \alpha$. Since $\mathfrak{D}_{p}, G=1,{ }_{z} N$ must be a $p$-group and $N / z N$ must be a $p$-group. Hence

$$
N / z N \subseteq \Omega_{1} z\left(\mathfrak{O}_{p} G / z N\right)
$$

VIII. Let e be a group theoretical property inherited by normal subgroups, direct products, and unions of towers of normal e-subgroups of a group. Then every hyper-egroup $G$ has

(1) normal subgroups $N_{1}$ and $Z_{1}$ such that

(a) $Z_{1} \circ G$ centralizes $N_{1} \circ G$,

(b) if the normal subgroup $X$ of $G$ has the property that $X \circ G$ centralizes $N_{1} \circ G$ then $X \subseteq Z_{1}$,

(c) $Z_{1} \subseteq N_{1}$ and $N_{1} / Z_{1}$ is an e-group,

(2) normal subgroups $N_{2}$ and $Z_{2}$ such that

(a) every subgroup of $Z_{2}$ permutes with every subgroup of $N_{2}$,

(b) if the normal subgroup $X$ of $G$ has the property that every subgroup of $X$ permutes with every subgroup of $N_{2}$, then $X \subseteq Z_{2}$,

(c) $Z_{2} \subseteq N_{2}$ and $N_{2} / Z_{2}$ is an e-group,

(3) normal subgroups $N_{3}$ and $Z_{3}$ depending on the choice of a group $\mathfrak{A}$ of automorphisms of $G$ containing all inner automorphisms such that

(a) every $\mathfrak{A}$-composition factor in $Z_{3}$ is centralized by $N_{3}$,

(b) if the normal subgroup $X$ of $G$ has the property that every $\mathfrak{A}$-composition factor of $G$ in $X$ centralizes every $\mathfrak{A}$-composition factor of $G$ in $N_{3}$, then $X \subseteq Z_{3}$,

(c) $Z_{3} \subseteq N_{3}$ and $N_{3} / Z_{3}$ is an e-group. 
Proof. Let $\nu$ be the set of all normal subgroups of $G$ and define $\alpha$ as in 3.I so that (1)-(6) are satisfied. The centralizer functions $\mathfrak{\complement}_{G}[f], \mathfrak{P}$, and $\mathfrak{\Re}$ on $\nu$ as described in 5.4.I, 5.2.II and III have the right properties to ensure the existence of a subgroup $A$ as in the main theorem. Since the union of a tower of normal $e$-subgroups of $G / A$ is an $e$-group, there will exist a normal subgroup $N$ with the properties required in the main theorem. By the theorem and the definition of the centralizer function $c$ to be used $Z_{i}=c_{0} N$ and $N_{i}=N$ have the properties (a), (b), (c).

7. Excursion on $[\nu, \alpha]-\delta$-immersion. Proposition 4 below will clarify the role of the hypothesis about $R$ in the main theorem. Let $\nu$ always satisfy (1) and (2). For $\nu$-subgroups $A \subseteq Q$ and subgroup theoretical properties $\alpha$ and $\delta$ on $\nu$ we define: $Q$ is $[v, \alpha]$ - $\delta$-immersed over $A$ in $G$, or $Q[\nu, \alpha]-\delta G$ over $A$, if

(12) $A \subseteq K \in \nu,(K,\langle K, Q\rangle, G) \notin \delta$ implies the existence of a $\nu$-subgroup $L$ with $K \subset L \subseteq\langle K, Q\rangle$ and $(K, L, G) \in \alpha$.

We denote by 1 the subgroup theoretical property $\{(X, X, G) \mid X \in \nu\}$. Then $[\nu, \alpha]$-1-immersion over 1 amounts to $[\nu, \alpha]$-immersion as defined in $\S 1$. If $1 \subseteq \delta$ then every $[\nu, \alpha]$-immersed $\nu$-subgroup is also $[\nu, \alpha]$ - $\delta$-immersed (over 1 ). We shall have to consider the following conditions on $\delta$ and $\alpha$ :

(13) $X, Y \in \nu, X \subseteq Y,(X, G, G) \in \delta$ implies $(Y, G, G) \in \delta$.

(14) If $K$ is a $\nu$-subgroup and if $\mathfrak{M}$ is a set of $\nu$-subgroups $X$ with $(K,\langle K, X\rangle, G)$ $\in \delta$, then $(K,\langle K, \mathfrak{M}\rangle, G) \in \delta$.

(15) $X \subseteq K \in \nu,(X, Y, G) \in \alpha$ implies $(K,\langle K, Y\rangle, G) \in \alpha$.

Note that (15) is satisfied whenever $\alpha$ is derived from a group theoretical property that is inherited by epimorphic images.

Proposition 3. (See R. Baer [2, Proposition 1.1]). Let $A \subseteq Q$ be v-subgroups.

(1) If $Q$ is $[\nu, \alpha]$ - $\delta$-immersed over $A$ and if the $\nu$-subgroup $B$ contains $A$, then $\langle Q, B\rangle$ is $[\nu, \alpha]$ - $\delta$-immersed over $B$.

(2) If $P$ is $[\nu, \alpha]$-1-immersed over $A$ and $Q$ is $[\nu, \alpha]$ - $\delta$-immersed over $P \subseteq Q$, then $Q$ is $[\nu, \alpha]$ - $\delta$-immersed over $A$.

(3) Suppose 5.1(b) and (14). If $\mathfrak{M}$ is a set of $\nu$-subgroups $X[\nu, \alpha]$ - $\delta$-immersed over $A$, then $\langle\mathfrak{M}\rangle$ is $[v, \alpha]$ - $\delta$-immersed over $A$.

(4) One may substitute $[\nu, \alpha]-\delta$ for $[\nu, \alpha]$ in the lemma of $\S 2$ if $\delta$ meets requirement (6) (with $\alpha$ replaced by $\delta$ ).

Proof. (1) and (3) are immediate applications of the definition of immersion.With regard to (2), let $K$ be a $\nu$-subgroup with $A \subseteq K$ and $(K,\langle K, Q\rangle, G) \notin \delta$. Assume first that $P \subseteq K$ or equivalently that $(K,\langle K, P\rangle, G) \notin 1$. Since $P$ is $[\nu, \alpha]-1-$ immersed over $A$, there exists a $\nu$-subgroup $L$ with $K \subset L \subseteq\langle K, P\rangle \subseteq\langle K, Q\rangle$ and $(K, L, G) \in \alpha$ as desired. If however $P \subseteq K$ then we may use that $Q$ is $[\nu, \alpha]-\delta$ immersed over $P$.-To verify (4) define $J$ as in the proof of the lemma. If $(J,\langle J, R\rangle, G) \in \delta$, then $(J \cap S, S, G) \in \delta$ by the implication (iii) $\rightarrow$ (i) in (6). But $J \cap S=K \cap S$, hence $(K,\langle K, S\rangle, G) \in \delta$ by (6). Therefore we may assume $(J,\langle J, R\rangle, G) \in \delta$ and using $R[\nu, \alpha]-\delta G$ proceed as in the lemma. 
We define the $[\nu, \alpha]-\delta$-hypercenter $\mathfrak{h}_{[v, \alpha]-\delta} G$ of $G$ to be the subgroup generated by all $[\nu, \alpha]$ - $\delta$-immersed $\nu$-subgroups (over 1) (see R. Baer [2]). Assuming 5.1(b) and (14) we conclude from Proposition $3(3)$ that $\mathfrak{h}_{[v, \alpha]-\delta} G$ is $[\nu, \alpha]-\delta$-immersed. In particular, since $\delta=1$ satisfies $(14), \mathfrak{h}_{[v, \alpha]} G$ is a $[\nu, \alpha]$-immersed $\nu$-subgroup whenever 5.1(b) holds.

Proposition 4. Let $v$ satisfy 5.1(b) and suppose that the subgroup theoretical properties $\delta$ and a meet requirements (13) and (15). Then the following statements are equivalent:

(a) There exists a $\nu$-subgroup $R$ with $R[\nu, \alpha] G$ and $(R, G, G) \in \delta$.

(b) $\left(\mathfrak{h}_{[v, \alpha]} G[\nu, \alpha] G\right.$ and $)\left(\mathfrak{h}_{[v, \alpha]} G, G, G\right) \in \delta$.

(c) $G[\nu, \alpha]-\delta G$.

Proof. The equivalence of (a) and (b) follows from 5.1(b) and (13). Assume (b) and let $H=\mathfrak{h}_{[v, \alpha]} G$. Let $K$ be a $\nu$-subgroup with $(K, G, G) \notin \delta$. By (b) and (13), $H \nsubseteq K$ so that $K \subset\langle K, H\rangle$. But $H[\nu, \alpha] G$ guarantees the existence of a $\nu$-subgroup $L$ with $K \subset L \subseteq\langle K, H\rangle$ and $(K, L, G) \in \alpha$. We proved (c). Conversely, assume $G[\nu, \alpha]-\delta G$ and by way of contradiction that $(H, G, G) \notin \delta$. Then there exists a $\nu$-subgroup $L \subset H$ with $(H, G, G) \in \alpha$. This implies that $L$ is $[\nu, \alpha]$-immersed over $H$, since for any $\nu$-subgroup $K$ with $H \subseteq K \subset\langle K, L\rangle$ we have $\langle K, L\rangle \in \nu$ with $K \subset\langle K, L\rangle \subseteq\langle K, L\rangle$ and $(K,\langle K, L\rangle, G) \in \alpha$ by (15). Now Proposition 3(2) yields that $L$ is $[\nu, \alpha]$-immersed and is hence contained in $H=\mathfrak{h}_{[v, \alpha]} G$, a contradiction, since $H \subset L$. Therefore $(H, G, G) \in \delta$, and the proof is complete.

\section{REFERENCES}

1. R. Baer, Polyminimaxgruppen, Math. Ann. 175 (1968), 1-43.

2. - Principal factors, maximal subgroups and conditional identities of finite groups, Illinois J. Math. 13 (1969), 1-52.

3. W. Feit and J. G. Thompson, Solvability of groups of odd order, Pacific J. Math. 13 (1963), 775-1029.

4. P. Hall and G. Higman, The p-length of a p-soluble group and reduction theorems for Burnside's problem, Proc. London Math. Soc. 6 (1956), 1-42.

5. U. Schoenwaelder, Centralizers of abelian, normal subgroups of hypercyclic groups, Pacific J. Math. (to appear).

6. M. Suzuki, Structure of a group and the structure of its lattice of subgroups, Ergebnisse der Mathematik und ihrer Grenzgebiete, Heft 10, Springer-Verlag, Berlin, 1956.

UNIVERSITY OF MisSOURI,

ST. Louis, Missouri 